OPEN ACCESS

Edited by:

Jeffrey G. Edwards,

Brigham Young University,

United States

Reviewed by:

John Neumaier,

University of Washington,

United States

Marc Fakhoury,

Lebanese American University,

Lebanon

*Correspondence:

Fereshteh S. Nugent

fereshteh.nugent@usuhs.edu

Received: 01 April 2021 Accepted: 29 April 2021

Published: 28 May 2021

Citation:

Shepard RD and Nugent FS (2021) Targeting Endocannabinoid Signaling in the Lateral Habenula as an Intervention to Prevent Mental IIInesses Following Early Life Stress:

A Perspective.

Front. Synaptic Neurosci. 13:689518. doi: 10.3389/fnsyn.2021.689518

\section{Targeting Endocannabinoid Signaling in the Lateral Habenula as an Intervention to Prevent Mental Illnesses Following Early Life Stress: A Perspective}

\author{
Ryan D. Shepard and Fereshteh S. Nugent*
}

Department of Pharmacology, F. Edward Hebert School of Medicine, Uniformed Services University of the Health Sciences, Bethesda, MD, United States

Adverse events and childhood trauma increase the susceptibility towards developing psychiatric disorders (substance use disorder, anxiety, depression, etc.) in adulthood. Although there are treatment strategies that have utility in combating these psychiatric disorders, little attention is placed on how to therapeutically intervene in children exposed to early life stress (ELS) to prevent the development of later psychopathology. The lateral habenula $(\mathrm{LHb})$ has been a topic of extensive investigation in mental health disorders due to its prominent role in emotion and mood regulation through modulation of brain reward and motivational neural circuits. Importantly, rodent models of ELS have been shown to promote LHb dysfunction. Moreover, one of the potential mechanisms contributing to LHb neuronal and synaptic dysfunction involves endocannabinoid (eCB) signaling, which has been observed to critically regulate emotion/mood and motivation. Many pre-clinical studies targeting eCB signaling suggest that this neuromodulatory system could be exploited as an intervention therapy to halt maladaptive processes that promote dysfunction in reward and motivational neural circuits involving the LHb. In this perspective article, we report what is currently known about the role of eCB signaling in $\mathrm{LHb}$ function and discuss our opinions on new research directions to determine whether the eCB system is a potentially attractive therapeutic intervention for the prevention and/or treatment of ELS-associated psychiatric illnesses.

Keywords: CB1R, early life stress, endocannabinoids, FAAH, lateral habenula, LHb, MAGL

\section{INTRODUCTION}

Early life stress (ELS) and childhood trauma have been recognized as critical risk factors that increase the probability of developing psychiatric disorders in both clinical observations and animal models (Heim and Nemeroff, 2002; Taylor, 2010; Targum and Nemeroff, 2019). Given our incomplete understanding of the neurobiological underpinnings and mental outcomes following ELS, as well as the wide array of maladaptive alterations in neural circuits induced by ELS, the development of efficacious interventional therapy has been hampered. At the time of writing this perspective, the coronavirus (COVID-19) pandemic 
has fundamentally changed societal norms with detrimental economic impact leading to increased psychological and physical stress resulting from a multitude of factors such as unemployment, social isolation and homelessness (Salari et al., 2020). Importantly, one of the deleterious effects of the COVID-19 pandemic has been the closure of schools resulting in social isolation of children from their peers and disproportional access to educational resources, which are especially lacking in minority communities and children with behavioral/cognitive deficits (Wong et al., 2020). In addition, there have been concerns of possible increased child maltreatment and neglect, as well as food and housing insecurity due to pandemic shutdowns across the globe (Humphreys et al., 2020). As such, growing epidemiological evidence suggests that adolescents living through the pandemic are potentially at a greater risk for depression and anxiety (Gotlib et al., 2020; Guessoum et al., 2020). Therefore, the issue of the consequences of ELS on the future well-being of children, now more than ever, are at the forefront of public health and further necessitates a complete understanding of the etiology of behavioral disorders associated with ELS and the identification of potential novel interventional therapeutic targets.

In conjunction with efforts aimed at increasing our knowledge of the molecular and cellular alterations that perturb the central nervous system (CNS) function in mental health disorders, identifying critical brain regions and neural circuits has remained of high importance. Stress-induced dysfunction in a number of brain regions following ELS has been linked to stress-related disorders including the amygdala (Malter Cohen et al., 2013), prefrontal cortex (Herzberg and Gunnar, 2020), and ventral tegmental area (VTA; Shepard and Nugent, 2020). Although these structures have been extensively studied, evidence now suggests that the lateral habenula (LHb), an evolutionarily conserved epithalamic structure, is a critical brain region that is involved in depression, substance use disorder, anxiety, and sleep disorders ( $\mathrm{Hu}$ et al., 2020). Earlier studies from our lab and others have demonstrated that rodent models of ELS promote $\mathrm{LHb}$ dysfunction through increased LHb neuronal excitability and altered synaptic transmission (Tchenio et al., 2017; Authement et al., 2018; Langlois et al., 2020; Simmons et al., 2020). Moreover, our longitudinal studies have demonstrated that these ELS-induced alterations are potentially long-lasting and span across multiple stages of development (Shepard et al., 2018b; Langlois et al., 2020).

\section{MATERIALS AND METHODS}

All experiments were conducted with approval from the Uniformed Services University (USU) Institutional Animal Care and Use Committee (IACUC) and guidelines from the National Institutes of Health (NIH) for the care and use of laboratory animals.

\section{Maternal Deprivation and LHb Microdissection}

Maternal deprivation (MD) was carried out as described previously (Authement et al., 2018; Shepard et al., 2018a,b,
2020; Simmons et al., 2020). At P9, male pups (Charles River) were isolated from the dam as a group in a separate room to avoid localization from other animals in the main housing area. MD pups were kept together and isolated from the dam for a total of $24 \mathrm{~h}$ before being returned to the home cage with the dam and remaining pups serving as the control group (nonmaternally deprived, non-MD rats). Rats were kept with the dam until P28 where they were weaned into dyads based on stress conditions (non-MD vs. MD). Between postnatal days 70-80, adult male rats were anesthetized with isoflurane and decapitated by guillotine. Brains were quickly dissected in ice-cold artificial cerebrospinal fluid (ACSF) containing (in $\mathrm{mM}$ ): $126 \mathrm{NaCl}$, $21.4 \mathrm{NaHCO}_{3}, 2.5 \mathrm{KCl}, 1.2 \mathrm{NaH}_{2} \mathrm{PO}_{4}, 2.4 \mathrm{CaCl}_{2}, 1.00 \mathrm{MgSO}_{4}$, 11.1 glucose, 0.4 ascorbic acid, saturated with $95 \% \mathrm{O}_{2}-5 \% \mathrm{CO}_{2}$. Sagittal brain sections were taken at $300 \mu \mathrm{m}$ and the LHb was grossly dissected from each as done previously (Authement et al., 2018). LHb was visualized by the slice containing both the stria medullaris and the fasciculus retroflexus.

\section{Western Blot}

WBs on LHb tissues were conducted as described previously (Authement et al., 2018). Twenty microgram of LHb protein lysates were loaded into precast polyacrylamide gels and separated by electrophoresis. Following transfer to nitrocellulose membranes, antibodies targeted against MAGL (1:500, Millipore ABN1000), FAAH (1:500, Abcam ab54615), GAPDH (1:1,000, Abcam ab9485) and vinculin (1:1,000, Abcam ab129002). HRP-linked secondary antibodies targeting either rabbit $(1: 2,000$, Cell Signaling) or mouse (1:2,000, Cell Signaling) were used. Data was analyzed using ImageJ. MAGL and FAAH expression levels were normalized to the levels of either GAPDH or vinculin. Summary data is reported as a fold change and is normalized to the non-MD group.

\section{Statistical Analysis}

Values for WB experiments are presented as means \pm SEM. Statistical significance was determined using unpaired Student's $t$-test and was set at a level of $p<0.05$. Due to a smaller sample size, the Shapiro-Wilk test was used to assess normality. Statistical analyses were performed using GraphPad Prism.

\section{The Lateral Habenula: a Lynchpin for the Development of Psychiatric Disorders?}

The habenula (both medial and lateral divisions) together with the stria medularis and pineal gland (Roman et al., 2020) make up the epithalamus which is conserved from humans down to teleosts indicating a critical evolutionary role (Sutherland, 1982). Specifically, the LHb functions as an "anti-reward" structure which is activated in response to aversion and negative reward (Hikosaka, 2010). Serving as a critical hub for incoming afferents projecting from forebrain and limbic regions (Hikosaka et al., 2008; Hu et al., 2020; Roman et al., 2020), the primarily glutamatergic pathways from the LHb suppress the release of monoamines such as dopamine (DA) and serotonin from the VTA (Ji and Shepard, 2007; Matsumoto and Hikosaka, 2007; Hong et al., 2011) and dorsal raphe nucleus (DRN; Pasquier et al., 1976; Varga et al., 2003), respectively, through their 
direct and indirect (GABAergic neurons of the rostromedial tegmental area, RMTg) projections. However, recent evidence suggests the existence of possible GABAergic interneurons in the LHb that could also provide local inhibitory signaling (Zhang et al., 2018; Flanigan et al., 2020; Webster et al., 2020; Li et al., 2021). It is important to note that LHb glutamatergic neurons are not homogenous and differ with respect to their physiological firing patterns, the expression of a variety of neuropeptidergic receptors, their downstream projections, and the inputs that they receive (Wallace et al., 2020). Studies have suggested that dysregulation of LHb function is involved in the pathophysiology of a variety of mental disorders (Lecca et al., 2014; Hu et al., 2020). Most notably, LHb hyperactivity has been consistently found in both clinical and animal models of depression (Proulx et al., 2014; Browne et al., 2018; Nuno-Perez et al., 2018; Yang et al., 2018; Cerniauskas et al., 2019; Gold and Kadriu, 2019). While LHb hyperactivity associated with depressive phenotypes have been found to occur uniformly across all LHb neuronal subpopulations ( $\mathrm{Li}$ et al., 2013; Authement et al., 2018; Shepard et al., 2018b; Yang et al., 2018; Simmons et al., 2020), some studies suggest projection- and input-specific LHb subcircuit dysfunction in depression (Li et al., 2011; Cerniauskas et al., 2019). For example, the enhanced release of glutamate at glutamatergic synapses onto VTA-projecting $\mathrm{LHb}$ neurons correlates with learned helplessness behaviors in rats ( $\mathrm{Li}$ et al., 2011) suggesting that depression-related hyperactivity in specific LHb subpopulations may arise from synaptic changes at distinct synaptic inputs to LHb subpopulations. Additionally, the entopeduncular (EP) nucleus (rodent homolog to the internal segment globus pallidus) co-releases glutamate/GABA to mediate aversion and "anti-reward" signaling. Antidepressants can alter changes in presynaptic glutamate/GABA release and thereby modulate LHb neuronal excitability (Shabel et al., 2012, 2014; Cerniauskas et al., 2019; Wallace et al., 2020). Consistently, chronic stress in mice increases the activity of VTA- but not DRN-projecting $\mathrm{LHb}$ neurons through enhanced presynaptic glutamate release from the EP, which underlies stress-induced increases in passive coping and reduced motivation, but not anxiety or anhedonia (Cerniauskas et al., 2019). Given that DA dysfunction is associated with ELS (Pruessner et al., 2004), our lab first focused on the effects of ELS on VTA DA function using an ELS model of MD in rats (Ellenbroek et al., 2005) in which rat pups are subjected to a single 24-h maternal separation (MS) from the dam. We demonstrated that MD induced VTA DA dysfunction through induction of GABAergic metaplasticity involving A-kinase anchoring scaffolding protein (AKAP150, also referred to AKAP79-the human equivalent to rodent AKAP150) signaling and histone deacetylases (HDACs). We showed that $\mathrm{MD}$ in juvenile rats selectively induces long-term depression (LTD) and shifts spike-timing-dependent plasticity (STDP) toward LTD at GABAergic synapses onto VTA DA neurons. This MD-induced metaplasticity involved epigenetic modifications to AKAP150 signaling that included an increase in HDAC2 and decreased histone acetylation which was reversible with HDAC inhibition (Authement et al., 2015; Shepard et al., 2018a, 2020; Shepard and Nugent, 2020). Taken together, this body of work demonstrates that MD alters GABAergic synaptic strength onto VTA DA neurons which potentially contributes to DA dysfunction in psychiatric disorders stemming from ELS.

Considering that the LHb regulates VTA DA signaling, we extended our studies to determine whether MD also perturbs $\mathrm{LHb}$ function. Studies from our lab using $\mathrm{MD}$ and others using maternal separation (MS; Tchenio et al., 2017) or the limited bedding and nesting models of ELS (Bolton et al., 2018) demonstrated that ELS can promote LHb hyperactivity (Authement et al., 2018; Shepard et al., 2018b; Simmons et al., 2020). Mechanistically, we have shown that LHb neurons in adolescent rats that underwent MD stress are hyperexcitable partly due to a shift in synaptic excitation and inhibition (E/I) balance towards excitation, as well as downregulation of small conductance (SK2) potassium channels and increased protein kinase (PKA) activity, resulting in induction of an intrinsic plasticity in LHb neurons. On the other hand, MS stress in mice decreases postsynaptic $G_{A B A} R$ signaling arising from entopeduncular nucleus GABAergic inputs which then contributes to MS-induced LHb hyperexcitability in adult mice (Tchenio et al., 2017). Given the epidemiological evidence for the increased predisposition towards developing psychiatric conditions of children subjected to early trauma, we also aimed to determine whether MD-induced LHb hyperactivity persists throughout adolescence into adulthood. Indeed, we have demonstrated persistent maladaptive alterations in $\mathrm{LHb}$ neuronal and synaptic function by MD from early adolescence (Authement et al., 2018; Shepard et al., 2018b; Simmons et al., 2020) into adulthood (Langlois et al., 2020). We also found that one of the possible mechanisms contributing to synaptic dysfunction in the LHb during early adolescence may be related to the potential impairment of eCB signaling (Authement et al., 2018) which we hypothesize partly contributes to the long-lasting ELS-induced LHb hyperexcitability.

\section{eCB Signaling: a Key Player in Synaptic Regulation}

Unlike neurotransmitters and neuropeptides, eCBs are lipids that are synthesized on-demand in an activity-dependent manner. The two major constituent eCB signaling messengers are $\mathrm{N}$-arachidonoylethanolamine (anandamide; AEA) and 2-arachidonoylglycerol (2-AG) which primarily act on cannabinoid receptors 1 and 2 (CB1R and CB2R) as retrograde signals, although they can also act on transient receptor potential vanilloid receptor type 1 (TRPV1) as non-retrograde signals (Castillo et al., 2012). There is a great deal of evidence to support that both AEA and 2-AG are synthesized by enzymes such as $N$-acylphosphatidylethanolamine-phospholipase $\mathrm{D}$ (NAPE-PLD) and diacylglycerol lipase (DAGL), respectively, that are localized to postsynaptic neurons (Katona et al., 2006; Yoshida et al., 2006; Yoshino et al., 2011). Interestingly, eCB degradation enzymes are segregated spatially in that fatty acid amide hydrolase (FAAH, the main degradative enzyme for AEA) is principally found postsynaptically and monoacylglycerol lipase (MAGL, the enzyme responsible for 2-AG degradation) is found presynaptically. Given these differences in spatial organization, it is reasonable to assume that these enzymatic 
synthesis/degradation pathways can be distinctly modulated with cell type-and brain region-specificity to regulate neuronal activity, synaptic strength (balance and/or coordination of excitatory to inhibitory inputs), and plasticity. Although there are a wide variety of receptors that eCBs can target independently of CB1Rs/CB2Rs, such as TRPV channels and other orphan receptors (Cristino et al., 2020), we will be discussing our perspective on $\mathrm{eCB}$ signaling within the context of $\mathrm{CB} 1 \mathrm{R}$ engagement due to its high neuronal expression (Qureshi et al., 1998) and robust role in synaptic plasticity (Gerdeman and Lovinger, 2003; Castillo et al., 2012). We recommend the following in-depth reviews for the role of eCB signaling in synaptic function and plasticity (Castillo et al., 2012; Augustin and Lovinger, 2018; Zou and Kumar, 2018; Cristino et al., 2020).

CB1Rs are $\mathrm{G}_{\mathrm{i} / \mathrm{o}}$-protein-coupled receptors (GPCRs) which once activated decrease the release of neurotransmitter presynaptically through a variety of mechanisms including decreased cyclic adenosine monophosphate (cAMP), membrane hyperpolarization through activation of potassium channels and decreased vesicular release of neurotransmitter via inhibition of voltage-gated calcium channels (VGCCs; Cristino et al., 2020). Although CB1Rs are primarily considered to be presynaptically localized, studies have also identified that CB1Rs can also function as autoreceptors when postsynaptically localized (Bacci et al., 2004). Lastly, others have identified that CB1Rs can also localize to glial cells, such as astrocytes (Navarrete and Araque, 2008). Moreover, depending on the localization of CB1Rs at distinct presynaptic inputs, eCBs are poised to differentially affect the strength of synaptic transmission in a cell-type and circuit-dependent manner. Importantly, eCBs are developmentally regulated and their levels fluctuate during critical developmental windows (Meyer et al., 2018). Not surprisingly, disease-based alterations in the neural function and behaviors involving eCB signaling have been implicated, making the eCB system a potential therapeutic target. Given the complexity of the $\mathrm{eCB}$ system that also includes several eCB-related mediators, their enzymes, and their molecular targets, the classical eCB signaling has been expanded to an "-ome"; the endocannabidiome (Cristino et al., 2020).

\section{ELS Potentially Impairs eCB Signaling in the LHb}

Studies on LHb physiology have demonstrated that eCB signaling profoundly controls LHb synaptic plasticity, neuronal activity, and associated behaviors (Valentinova and Mameli, 2016; Park et al., 2017; Authement et al., 2018; Berger et al., 2018). Potential alterations in eCB-mediated regulation of LHb neurons could contribute to LHb dysfunction associated with anhedonia, as well as motivational and social deficits in depression and other stressrelated disorders.

In fact, CB1Rs are shown to be highly expressed in the $\mathrm{LHb}$, specifically in presynaptic axon terminals, postsynaptic dendrites and glia (Berger et al., 2018). Intra-LHb manipulations of CB1Rs, has been shown to bi-directionally affect stress coping strategies where upon LHb CB1R blockade, rats display a more proactive behavioral style over avoidance (i.e., rats are more likely to explore and interact with a novel conspecific) whereas
LHb CB1R activation increases avoidant behaviors (Berger et al., 2018). These data suggest that LHb eCB signaling has profound effects on motivational and social behaviors which could be mediated by eCB-mediated synaptic modifications in the LHb. The two forms of synaptic plasticity (long-term potentiation, LTP, and LTD) are both subject to eCB modulation (Castillo et al., 2012), although eCBs mostly depress synaptic transmission (through a decrease in presynaptic neurotransmitter release), inhibit LTP and promote the induction of a presynaptic LTD at both glutamatergic and GABAergic synapses (Castillo et al., 2012). In response to a traditional LTD protocol (low frequency stimulation), or upon activation of group I metabotropic glutamate receptor (mGluR I), LHb neurons can express an eCB-mediated presynaptic LTD at glutamatergic synapses (Valentinova and Mameli, 2016; Park et al., 2017). Importantly, a strong stressor (delivering unpredictable tail shocks while rats are restrained) is sufficient to block eCB-mediated glutamatergic LTD in LHb neurons, potentially enhancing the excitatory drive onto $\mathrm{LHb}$ neurons following this acute stress. The stressinduced impairment of eCB-mediated LTD is recovered by inhibition of CamKIIa (an important LHb molecular substrate in behavioral depression; Park et al., 2017). Interestingly, mGluR activation also triggers LTD at GABAergic synapses through PKC-dependent reduction of $\beta 2$-containing $\mathrm{GABA}_{\mathrm{A}} \mathrm{Rs}$ in $\mathrm{LHb}$ neurons suggesting that $\mathrm{LHb}$ mGluR signaling bidirectionally regulates $\mathrm{LHb}$ neuronal outputs through induction of mGluRdependent synaptic plasticity that could mediate opposing motivational behaviors (Valentinova and Mameli, 2016).

We also found that activation and inhibition of CB1Rs decreases and increases the probability of presynaptic GABA release in the $\mathrm{LHb}$, respectively, suggesting a possible presence of a tonic eCB signaling and persistent $\mathrm{CB} 1$ receptor activation in the regulation of GABAergic transmission in the $\mathrm{LHb}$ (Authement et al., 2018). Importantly, we have shown that activation of corticotropin-releasing factor (CRF)-CRFR1PKA signaling increases LHb excitability through selective suppression of presynaptic GABA release onto LHb neurons through retrograde $\mathrm{eCB}-\mathrm{CB} 1 \mathrm{R}$ signaling, as well as increases in intrinsic excitability of $\mathrm{LHb}$ neurons through PKA-dependent reduction of SK2 channels. More importantly, we observed blunted responses of $\mathrm{LHb}$ neurons to the excitatory effects of acute CRF signaling in LHb neurons due to MD-induced increases in PKA activity, possibly downregulating SK2 channels in LHb neurons (Authement et al., 2018).

Our longitudinal studies on the effects of $\mathrm{MD}$ on $\mathrm{LHb}$ function demonstrated the persistence of $\mathrm{MD}$-induced $\mathrm{LHb}$ hyperexcitability during development (Shepard et al., 2018b; Langlois et al., 2020). Interestingly, we have also observed potentially long-lasting elevations in eCB-2AG levels in the $\mathrm{LHb}$ as we found decreased MAGL (2-AG degrading enzyme) protein levels, as well as decreases in CB1R expression in the adult rat LHb (Figure 1). This is in line with the studies from knock-out $(\mathrm{KO})$ mice where either deletion of MAGL or chronic antagonism of MAGL can decrease CB1R expression in the CNS (Chanda et al., 2010; Schlosburg et al., 2014). Consistently, chronic unpredictable stress has been shown to alter eCB degradation and is associated with less CB1R binding 
A
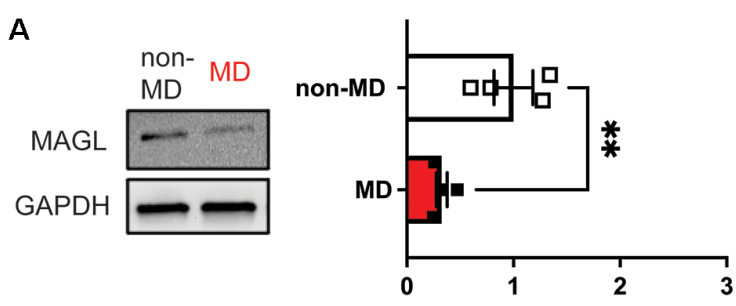

B
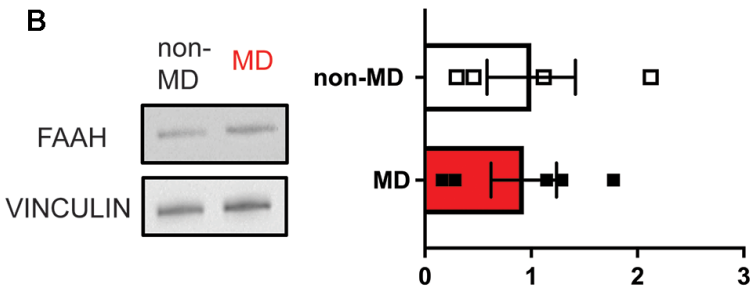

C
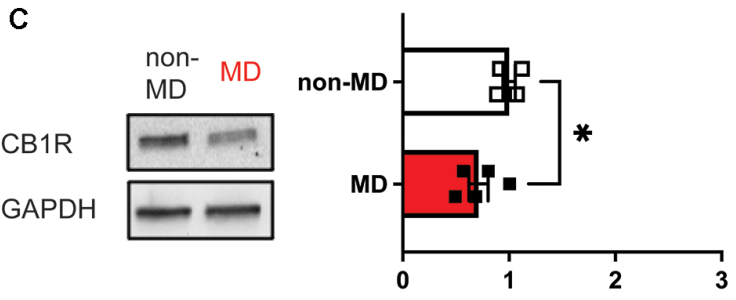

FIGURE 1 | Maternal deprivation (MD) decreases the abundance of both MAGL and CB1R in LHb whole protein lysates, but not FAAH in adulthood (Postnatal days 70-80) in response to ELS at Postnatal day 9. Bar graphs represent fold changes in the abundance of protein with respect to non-MD rats. (A) MD decreases total MAGL abundance (non-MD, $n=4$; MD, $n=4$; $t_{(6)}=3.543$ ). (B) MD does not significantly affect FAAH abundance (non-MD, $\left.n=4 ; \mathrm{MD}, n=5 ; t_{(7)}=0.1360\right)$. (C) MD decreases CB1R abundance (non-MD, $n=4$; MD, $n=5 ; t_{(7)}=2.522$ ). ${ }^{*} p<0.05,{ }^{* *} p<0.01$ by unpaired $t$-test.

(Berger et al., 2018). Similarly, a recent study demonstrated that exposure to alcohol in rats decreases CB1R expression, but in contrast, increases MAGL with no change in FAAH. Interestingly, this study also found that activation of $\mathrm{eCB}$ signaling within the $\mathrm{LHb}$ is an analgesic and could reduce ethanol intake (Fu et al., 2021). Additionally, KO of MAGL can promote anxiety- and pro-depressive behaviors (Imperatore et al., 2015) providing additional evidence for a critical role of eCB modulation of neural circuits that regulate stress-related behaviors. Given that adult $\mathrm{MD}$ rats show an imbalance of excitation and inhibition towards excitation with LHb hyperactivity (Shepard et al., 2018b; Langlois et al., 2020), we assume that this could also contribute to an elevation in $\mathrm{eCB}$ 2-AG production. Although we show the interaction between CRF neuromodulatory systems with eCB signaling within the adolescent rat $\mathrm{LHb}$ following $\mathrm{MD}$, whether MD-induced dysregulation of CRF signaling persists into adulthood and affects eCB-mediated regulation of $\mathrm{LHb}$ activity is unclear. Given that MD-induced CRF dysregulation seems to promote intrinsic LHb excitability and that eCB-CB1R signaling decreases both presynaptic glutamate and GABA release and mediates glutamatergic LTD in $\mathrm{LHb}$, we predict that MD may decrease 2-AG degradation by $\mathrm{MD}$-induced decreases in presynaptic MAGL specific to presynaptic GABAergic inputs to the $\mathrm{LHb}$ while selective $\mathrm{MD}$-induced downregulation of CB1Rs in presynaptic glutamatergic terminals within the $\mathrm{LHb}$ could decrease eCB-mediated suppression of glutamate release as well as inhibit eCB-mediated LTD in LHb neurons. Interestingly, a recent study demonstrated that most cannabinoids suppress the reinforcing effects of optogenetic VTA DA neuron self-stimulation in mice, suggesting that cannabinoid receptor activation, in general, attenuates VTA DA reward or could exert aversive effects. This study also shows that VTA GABA and glutamate neurons express CB1Rs while VTA DA neurons express CB2Rs (Humburg et al., 2021). Given that VTA GABA neurons also provide inhibitory GABAergic input to $\mathrm{LHb}$, it is possible that ELS-induced decreases in MAGL expression and persistent increases in eCB 2AG-CB1R-mediated suppression of VTA GABAergic input to the LHb promotes ELS-associated LHb hyperactivity. However, a technical limitation of our molecular data is the lack of cell-type and synapse-specific localizations of the enzyme and receptor which poses the question whether MD-induced downregulation of CB1R and MAGL occurs globally at all excitatory and inhibitory synaptic inputs and $\mathrm{LHb}$ neurons projecting to different downstream targets. Our studies show that MD increases the overall activity of LHb neurons and induces behavioral changes including increased immobility in the forced swim test, reduced sucrose preference, and decreased motivation for morphine self-administration (Shepard et al., 2018b; Langlois et al., 2020). Given that distinct LHb subcircuits may mediate specific behavioral phenotypes as shown following chronic stress model of depression in mice (Cerniauskas et al., 2019), future ELS research is necessary to investigate the precise contribution of ELS-induced hyperactivity of VTA-, RMTg- and DRN-projecting LHb neurons as well as eCB-mediated synaptic plasticity at specific synaptic inputs to the $\mathrm{LHb}$ in different behavioral deficits following ELS. Moreover, it is worthwhile to examine how different ELS models (MD, MS or LBN) affect stress neuromodulatory systems such as CRF (Authement et al., 2018) and kappa opioid receptor signaling (Simmons et al., 2020) within the LHb with their possible interaction with the $\mathrm{eCB}$ system in mediating motivational and anhedonic states associated with these ELS models as cell type and circuit-specific manner.

\section{DISCUSSION}

New advances in understanding neural circuits and the connectome have shaped our understanding of how discrete brain regions and circuits become dysregulated in psychiatric and neurological disorders. Given that $\mathrm{eCB}$ signaling plays a critical role in neuronal regulation across the CNS, greater emphasis needs to be placed on the role of eCB signaling with respect to specific inputs and brain structures. Advancements in mouse genetics and Cre-dependent manipulations of neuronal activity (optogenetics and DREADDs) and gene expression allow us to gain greater spatial and temporal control for cell typeand circuit-specific eCB neuromodulation and eCB-mediated 
behaviors. In addition to using preclinical animal models to study the role of eCB signaling, it is also important to understand whether pharmacological manipulation of eCB signaling in a clinical setting is a viable treatment method. Until recently, one problem with imaging the habenula was due to its small size which makes functional imaging a challenge (Lawson et al., 2013). Recently, using magnetic resonance imaging (MRI) with better resolution has made it possible to functionally image the human habenula (Strotmann et al., 2013, 2014). However, it is still not possible to reliably distinguish between the medial and lateral divisions in humans.

Recent legislative actions have now allowed the use of medical marijuana for the treatment of a variety of conditions which vary across the United States and countries around the world. However, one concern of the use of marijuana and other derivatives as treatment is their potential for abuse, as well as the potential for inducing schizophrenia and other psychiatric disorders (Bostwick, 2012; Chadwick et al., 2013). Therefore, a large number of clinical trials have both been conducted and are currently underway to determine whether targeting CB1R directly or affecting the degradation rate of eCBs through FAAH and MAGL inhibitors can be used in a wide variety of disorders ranging from anxiety to epilepsy (van Egmond et al., 2021); however, there is still a lack of clinical data to determine whether these compounds will be efficacious across the general population. In addition, there is greater concern over whether treatment in pediatrics and adolescents would be safe (Fontanella et al., 2021) considering the critical role of the eCB system in development (Meyer et al., 2018). Therefore, future drug development could potentially focus more so on targeting downstream eCB signaling from CB1Rs.

Advances in proteomic screenings have started to allow the identification of protein-protein interactions of receptor complexes. In fact, many receptors can exist in nature as receptor complexes where accessory proteins or auxiliary subunits can impact receptor trafficking, kinetics, and pharmacology (Maher et al., 2017). Already, these accessory proteins have been identified for VGCCs (Campiglio and Flucher, 2015), AMPARs (Kamalova and Nakagawa, 2021), nAChRs (Boulin et al., 2012), and most recently $\mathrm{GABA}_{\mathrm{A}}$ Rs (Castellano et al., 2020; Han et al., 2020). Indeed, targeting of auxiliary subunits has already succeeded with gabapentin being used for the treatment of epilepsy and pain. Currently, the transmembrane AMPAR regulatory protein (TARP) auxiliary subunit $\gamma 8$ is in clinical trials for the treatment of pain and epilepsy (Kato et al., 2016; Maher et al., 2017). Targeting receptor complexes offer an exciting opportunity for more precision-based pharmacology and to mitigate off-target effects associated with numerous drugs.

Like other receptors, CB1Rs are expressed ubiquitously in the CNS and targeting CB1R-associated proteins to modify downstream intracellular signaling could perhaps yield novel drug targets for development, such as cannabinoid receptor-interacting protein $1 \mathrm{a}$ and $1 \mathrm{~b}$ (CRIP1a and CRIP1b, respectively). For example, CRIP1a is a CB1R-specific accessory protein (Niehaus et al., 2007) which has been demonstrated to compete with b-arrestin binding which prevents desensitization and internalization of the receptor (Blume et al., 2017).
Additionally, CRIP1a can alter CB1R signaling by altering GPCR signaling pathways (Blume et al., 2016) and stopping CB1R-mediated closure of VGCCs (Niehaus et al., 2007). Within this context, it is perhaps possible that our MD-induced decreases in CB1R in adult rat LHb (Figure 1) is due to decreased CRIP1A which allows b-arrestin-mediated endocytosis of CB1Rs; however, this is pure speculation and would need to be verified by both CRIP1a expression, as well as biochemical studies demonstrating the association of both CRIP1a and CB1R in the $\mathrm{LHb}$. Therefore, further research regarding the role of CRIP1a and CRIP1b signaling in a cell type-and circuit-specific manner, in preclinical animal models would provide critical data on the physiological role of these accessory proteins as well as whether they are dysregulated in ELS animal models.

\section{CONCLUSION}

In summary, given the robust role of the $\mathrm{LHb}$ in psychiatric disorders as well as the role of CB1R-mediated regulation of $\mathrm{LHb}$ activity, targeting the $\mathrm{eCB}$ system for ELS-induced psychiatric disorders is a potential therapeutic option. Although a majority of the clinical data has been conducted in adults, greater observations to the role of eCB signaling in adolescents, as well as possible clinical administration of eCB-targeting compounds could provide data as to their safety and efficacy in younger individuals. Lastly, targeting receptor complexes (Rosenbaum et al., 2020) might be a more precise therapy that can reduce the likelihood of adverse off-target effects and mitigate the aforementioned concerns.

\section{DATA AVAILABILITY STATEMENT}

The original contributions presented in the study are included in the article, further inquiries can be directed to the corresponding author.

\section{ETHICS STATEMENT}

The animal study was reviewed and approved by Uniformed Services University IACUC committee.

\section{AUTHOR CONTRIBUTIONS}

FN and RS designed the experiments and wrote the manuscript. RS performed the experiments, analyzed the data, and prepared the figures. All authors contributed to the article and approved the submitted version.

\section{FUNDING}

This work was supported by the National Institutes of Health (NIH) - National Institute of Drugs of Abuse (NIDA) Grant\# R01 DA039533 to FN.

\section{ACKNOWLEDGMENTS}

The opinions and assertions contained herein are the private opinions of the authors and are not to be 
construed as official or reflecting the views of the Uniformed Services University of the Health Sciences

\section{REFERENCES}

Augustin, S. M., and Lovinger, D. M. (2018). Functional relevance of endocannabinoid-dependent synaptic plasticity in the central nervous system. ACS Chem. Neurosci. 9, 2146-2161. doi: 10.1021/acschemneuro.7b 00508

Authement, M. E., Kodangattil, J. N., Gouty, S., Rusnak, M., Symes, A. J., Cox, B. M., et al. (2015). Histone deacetylase inhibition rescues maternal deprivation-induced GABAergic metaplasticity through restoration of AKAP signaling. Neuron 86, 1240-1252. doi: 10.1016/j.neuron.2015.05.024

Authement, M. E., Langlois, L. D., Shepard, R. D., Browne, C. A., Lucki, I., Kassis, H., et al. (2018). A role for corticotropin-releasing factor signaling in the lateral habenula and its modulation by early-life stress. Sci. Signal. 11:eaan6480. doi: 10.1126/scisignal.aan6480

Bacci, A., Huguenard, J. R., and Prince, D. A. (2004). Long-lasting self-inhibition of neocortical interneurons mediated by endocannabinoids. Nature 431, 312-316. doi: 10.1038/nature02913

Berger, A. L., Henricks, A. M., Lugo, J. M., Wright, H. R., Warrick, C. R., Sticht, M. A., et al. (2018). The lateral habenula directs coping styles under conditions of stress via recruitment of the endocannabinoid system. Biol. Psychiatry 84, 611-623. doi: 10.1016/j.biopsych.2018.04.018

Blume, L. C., Leone-Kabler, S., Luessen, D. J., Marrs, G. S., Lyons, E., Bass, C. E., et al. (2016). Cannabinoid receptor interacting protein suppresses agonistdriven CB1 receptor internalization and regulates receptor replenishment in an agonist-biased manner. J. Neurochem. 139, 396-407. doi: 10.1111/jnc.13767

Blume, L. C., Patten, T., Eldeeb, K., Leone-Kabler, S., Ilyasov, A. A., Keegan, B. M., et al. (2017). Cannabinoid receptor interacting protein la competition with $\beta$-arrestin for CB1 receptor binding sites. Mol. Pharmacol. 91, 75-86. doi: $10.1124 / \mathrm{mol} .116 .104638$

Bolton, J. L., Ruiz, C. M., Rismanchi, N., Sanchez, G. A., Castillo, E., Huang, J., et al. (2018). Early-life adversity facilitates acquisition of cocaine self-administration and induces persistent anhedonia. Neurobiol. Stress 8, 57-67. doi: 10.1016/j. ynstr.2018.01.002

Bostwick, J. M. (2012). Blurred boundaries: the therapeutics and politics of medical marijuana. Mayo Clin. Proc. 87, 172-186. doi: 10.1016/j.mayocp.2011.10.003

Boulin, T., Rapti, G., Briseño-Roa, L., Stigloher, C., Richmond, J. E., Paoletti, P., et al. (2012). Positive modulation of a Cys-loop acetylcholine receptor by an auxiliary transmembrane subunit. Nat. Neurosci. 15, 1374-1381. doi: $10.1038 / \mathrm{nn} .3197$

Browne, C. A., Hammack, R., and Lucki, I. (2018). Dysregulation of the lateral habenula in major depressive disorder. Front. Synaptic Neurosci. 10:46. doi: $10.3389 /$ fnsyn.2018.00046

Campiglio, M., and Flucher, B. E. (2015). The role of auxiliary subunits for the functional diversity of voltage-gated calcium channels. J. Cell. Physiol. 230, 2019-2031. doi: 10.1002/jcp.24998

Castellano, D., Shepard, R. D., and Lu, W. (2020). Looking for novelty in an "Old" receptor: recent advances toward our understanding of GABARs and their implications in receptor pharmacology. Front. Neurosci. 14:616298. doi: $10.3389 /$ fnins.2020.616298

Castillo, P. E., Younts, T. J., Chávez, A. E., and Hashimotodani, Y. (2012). Endocannabinoid signaling and synaptic function. Neuron 76, 70-81. doi: 10.1016/j.neuron.2012.09.020

Cerniauskas, I., Winterer, J., de Jong, J. W., Lukacsovich, D., Yang, H., Khan, F., et al. (2019). Chronic stress induces activity, synaptic and transcriptional remodeling of the lateral habenula associated with deficits in motivated behaviors. Neuron 104, 899-915. doi: 10.1016/j.neuron.2019. 09.005

Chadwick, B., Miller, M. L., and Hurd, Y. L. (2013). Cannabis use during adolescent development: susceptibility to psychiatric illness. Front. Psychiatry 4:129. doi: 10.3389/fpsyt.2013.00129

Chanda, P. K., Gao, Y., Mark, L., Btesh, J., Strassle, B. W., Lu, P., et al. (2010). Monoacylglycerol lipase activity is a critical modulator of the tone and integrity of the endocannabinoid system. Mol. Pharmacol. 78, 996-1003. doi: $10.1124 / \mathrm{mol} .110 .068304$ or the Department of Defense or the Government of the United States.

Cristino, L., Bisogno, T., and Di Marzo, V. (2020). Cannabinoids and the expanded endocannabinoid system in neurological disorders. Nat. Rev. Neurol. 16, 9-29. doi: 10.1038/s41582-019-0284-z

Ellenbroek, B. A., Derks, N., and Park, H.-J. (2005). Early maternal deprivation retards neurodevelopment in Wistar rats. Stress 8, 247-257. doi: $10.1080 / 10253890500404634$

Flanigan, M. E., Aleyasin, H., Li, L., Burnett, C. J., Chan, K. L., LeClair, K. B., et al. (2020). Orexin signaling in GABAergic lateral habenula neurons modulates aggressive behavior in male mice. Nat. Neurosci. 23, 638-650. doi: 10.1038/s41593-020-0617-7

Fontanella, C. A., Steelesmith, D. L., Brock, G., Bridge, J. A., Campo, J. V., and Fristad, M. A. (2021). Association of cannabis use with self-harm and mortality risk among youths with mood disorders. JAMA Pediatr. 175, 377-384. doi: 10.1001/jamapediatrics.2020.5494

Fu, R., Tang, Y., Li, W., Ren, Z., Li, D., Zheng, J., et al. (2021). Endocannabinoid signaling in the lateral habenula regulates pain and alcohol consumption. Transl. Psychiatry 11:220. doi: 10.1038/s41398-021-01337-3

Gerdeman, G. L., and Lovinger, D. M. (2003). Emerging roles for endocannabinoids in long-term synaptic plasticity. Br. J. Pharmacol. 140, 781-789. doi: 10.1038/sj.bjp.0705466

Gold, P. W., and Kadriu, B. (2019). A major role for the lateral habenula in depressive illness: physiologic and molecular mechanisms. Front. Psychiatry 10:320. doi: 10.3389/fpsyt.2019.00320

Gotlib, I. H., Borchers, L. R., Chahal, R., Gifuni, A. J., Teresi, G. I., and Ho, T. C. (2020). Early life stress predicts depressive symptoms in adolescents during the COVID-19 pandemic: the mediating role of perceived stress. Front. Psychol. 11:603748. doi: 10.3389/fpsyg.2020.603748

Guessoum, S. B., Lachal, J., Radjack, R., Carretier, E., Minassian, S., Benoit, L., et al. (2020). Adolescent psychiatric disorders during the COVID-19 pandemic and lockdown. Psychiatry Res. 291:113264. doi: 10.1016/j.psychres.2020.113264

Han, W., Shepard, R. D., and Lu, W. (2020). Regulation of GABAARs by Transmembrane Accessory Proteins. Trends Neurosci. 44, 152-165. doi: 10.1016/j.tins.2020.10.011

Heim, C., and Nemeroff, C. B. (2002). Neurobiology of early life stress: clinical studies. Semin. Clin. Neuropsychiatry 7, 147-159. doi: 10.1053/scnp.2002.33127

Herzberg, M. P., and Gunnar, M. R. (2020). Early life stress and brain function: activity and connectivity associated with processing emotion and reward. NeuroImage 209:116493. doi: 10.1016/j.neuroimage. 2019.116493

Hikosaka, O. (2010). The habenula: from stress evasion to value-based decisionmaking. Nat. Rev. Neurosci. 11, 503-513. doi: 10.1038/nrn2866

Hikosaka, O., Sesack, S. R., Lecourtier, L., and Shepard, P. D. (2008). Habenula: crossroad between the basal ganglia and the limbic system. J. Neurosci. 28, 11825-11829. doi: 10.1523/JNEUROSCI.3463-08.2008

Hong, S., Jhou, T. C., Smith, M., Saleem, K. S., and Hikosaka, O. (2011). Negative reward signals from the lateral habenula to dopamine neurons are mediated by rostromedial tegmental nucleus in primates. J. Neurosci. 31, 11457-11471. doi: 10.1523/JNEUROSCI.1384-11.2011

$\mathrm{Hu}, \mathrm{H} ., \mathrm{Cui}, \mathrm{Y}$., and Yang, Y. (2020). Circuits and functions of the lateral habenula in health and in disease. Nat. Rev. Neurosci. 21, 277-295. doi: 10.1038/s41583020-0292-4

Humburg, B. A., Jordan, C. J., Zhang, H. Y., Shen, H., Han, X., Bi, G. H., et al. (2021). Optogenetic brain-stimulation reward: a new procedure to re-evaluate the rewarding versus aversive effects of cannabinoids in dopamine transporterCre mice. Addict. Biol. doi: 10.1111/adb.13005. [Epub ahead of print].

Humphreys, K. L., Myint, M. T., and Zeanah, C. H. (2020). Increased Risk for Family Violence During the COVID-19 Pandemic. Pediatrics 146:e20200982. doi: 10.1542/peds.2020-0982

Imperatore, R., Morello, G., Luongo, L., Taschler, U., Romano, R., De Gregorio, D. et al. (2015). Genetic deletion of monoacylglycerol lipase leads to impaired cannabinoid receptor CBR signaling and anxiety-like behavior. J. Neurochem. 135, 799-813. doi: 10.1111/jnc. 13267

Ji, H., and Shepard, P. D. (2007). Lateral Habenula Stimulation Inhibits Rat Midbrain Dopamine Neurons through a GABAA Receptor-Mediated 
Mechanism. J. Neurosci. 27, 6923-6930. doi: 10.1523/JNEUROSCI.0958 $-07.2007$

Kamalova, A., and Nakagawa, T. (2021). AMPA receptor structure and auxiliary subunits. J. Physiol. 599, 453-469. doi: 10.1113/JP278701

Kato, A. S., Burris, K. D., Gardinier, K. M., Gernert, D. L., Porter, W. J., Reel, J., et al. (2016). Forebrain-selective AMPA-receptor antagonism guided by TARP $\gamma-8$ as an antiepileptic mechanism. Nat. Med. 22, 1496-1501. doi: 10.1038/ nm. 4221

Katona, I., Urbán, G. M., Wallace, M., Ledent, C., Jung, K.-M., Piomelli, D., et al. (2006). Molecular composition of the endocannabinoid system at glutamatergic synapses. J. Neurosci. 26, 5628-5637. doi: 10.1523/JNEUROSCI. 0309-06.2006

Langlois, L. D., Berman, R. Y., Shepard, R. D., Simmons, S. C., Tsuda, M. C., Gouty, S., et al. (2020). Potentiation of glutamatergic synaptic transmission onto lateral habenula neurons following early life stress and intravenous morphine self-administration in rats. bioRxiv [Preprint]. doi: 10.1101/2020.12. 23.424217

Lawson, R. P., Drevets, W. C., and Roiser, J. P. (2013). Defining the habenula in human neuroimaging studies. NeuroImage 64, 722-727. doi: 10.1016/j. neuroimage.2012.08.076

Lecca, S., Meye, F. J., and Mameli, M. (2014). The lateral habenula in addiction and depression: an anatomical, synaptic and behavioral overview. Eur. J. Neurosci. 39, 1170-1178. doi: 10.1111/ejn. 12480

Li, B., Piriz, J., Mirrione, M., Chung, C., Proulx, C. D., Schulz, D., et al. (2011). Synaptic potentiation onto habenula neurons in the learned helplessness model of depression. Nature 470, 535-539. doi: 10.1038/nature09742

Li, J., Fan, R., Liu, X., Shen, X., Liu, X., and Zhao, H. (2021). The convergence of aversion and reward signals in individual neurons of the mice lateral habenula. Exp. Neurol. 339:113637. doi: 10.1016/j.expneurol.2021.113637

Li, K., Zhou, T., Liao, L., Yang, Z., Wong, C., Henn, F., et al. (2013). BCaMKII in lateral habenula mediates core symptoms of depression. Science 341, 1016-1020. doi: 10.1126/science.1240729

Maher, M. P., Matta, J. A., Gu, S., Seierstad, M., and Bredt, D. S. (2017). Getting a handle on neuropharmacology by targeting receptor-associated proteins. Neuron 96, 989-1001. doi: 10.1016/j.neuron.2017.10.001

Malter Cohen, M., Jing, D., Yang, R. R., Tottenham, N., Lee, F. S., and Casey, B. J. (2013). Early-life stress has persistent effects on amygdala function and development in mice and humans. Proc. Natl. Acad. Sci. U. S. A. 110, 18274-18278. doi: 10.1073/pnas.1310163110

Matsumoto, M., and Hikosaka, O. (2007). Lateral habenula as a source of negative reward signals in dopamine neurons. Nature 447, 1111-1115. doi: $10.1038 /$ nature 05860

Meyer, H. C., Lee, F. S., and Gee, D. G. (2018). The role of the endocannabinoid system and genetic variation in adolescent brain development. Neuropsychopharmacology 43, 21-33. doi: 10.1038/npp.2017.143

Navarrete, M., and Araque, A. (2008). Endocannabinoids mediate neuronastrocyte communication. Neuron 57, 883-893. doi: 10.1016/j.neuron.2008. 01.029

Niehaus, J. L., Liu, Y., Wallis, K. T., Egertová, M., Bhartur, S. G., Mukhopadhyay, S., et al. (2007). CB1 cannabinoid receptor activity is modulated by the cannabinoid receptor interacting protein CRIP 1a. Mol. Pharmacol. 72, 1557-1566. doi: 10.1124/mol.107.039263

Nuno-Perez, A., Tchenio, A., Mameli, M., and Lecca, S. (2018). Lateral habenula gone awry in depression: bridging cellular adaptations with therapeutics. Front. Neurosci. 12:485. doi: 10.3389/fnins.2018.00485

Park, H., Rhee, J., Lee, S., and Chung, C. (2017). Selectively impaired endocannabinoid-dependent long-term depression in the lateral habenula in an animal model of depression. Cell Rep. 20, 289-296. doi: 10.1016/j.celrep.2017. 06.049

Pasquier, D. A., Anderson, C., Forbes, W. B., and Morgane, P. J. (1976). Horseradish peroxidase tracing of the lateral habenular-midbrain raphe nuclei connections in the rat. Brain Res. Bull. 1, 443-451. doi: 10.1016/03619230(76)90114-3

Proulx, C. D., Hikosaka, O., and Malinow, R. (2014). Reward processing by the lateral habenula in normal and depressive behaviors. Nat. Neurosci. 17, 1146-1152. doi: 10.1038/nn.3779

Pruessner, J. C., Champagne, F., Meaney, M. J., and Dagher, A. (2004). Dopamine release in response to a psychological stress in humans and its relationship to early life maternal care: a positron emission tomography study using [11C]raclopride. J. Neurosci. 24, 2825-2831. doi: 10.1523/JNEUROSCI.342203.2004

Qureshi, J., Saady, M., Cardounel, A., and Kalimi, M. (1998). Identification and characterization of a novel synthetic cannabinoid CP 55,940 binder in rat brain cytosol. Mol. Cell. Biochem. 181, 21-27. doi: 10.1023/a:1006855504094

Roman, E., Weininger, J., Lim, B., Roman, M., Barry, D., Tierney, P., et al. (2020). Untangling the dorsal diencephalic conduction system: a review of structure and function of the stria medullaris, habenula and fasciculus retroflexus. Brain Struct. Funct. 225, 1437-1458. doi: 10.1007/s00429-020-02069-8

Rosenbaum, M. I., Clemmensen, L. S., Bredt, D. S., Bettler, B., and Strømgaard, K. (2020). Targeting receptor complexes: a new dimension in drug discovery. Nat. Rev. Drug Discov. 19, 884-901. doi: 10.1038/s41573-020-0086-4

Salari, N., Hosseinian-Far, A., Jalali, R., Vaisi-Raygani, A., Rasoulpoor, S., Mohammadi, M., et al. (2020). Prevalence of stress, anxiety, depression among the general population during the COVID-19 pandemic: a systematic review and meta-analysis. Global Health 16:57. doi: 10.1186/s12992-020 -00589-w

Schlosburg, J. E., Kinsey, S. G., Ignatowska-Jankowska, B., Ramesh, D., Abdullah, R. A., Tao, Q., et al. (2014). Prolonged monoacylglycerol lipase blockade causes equivalent cannabinoid receptor type 1 receptor-mediated adaptations in fatty acid amide hydrolase wild-type and knockout mice. J. Pharmacol. Exp. Ther. 350, 196-204. doi: 10.1124/jpet.114.212753

Shabel, S. J., Proulx, C. D., Piriz, J., and Malinow, R. (2014). Mood regulation. GABA/glutamate co-release controls habenula output and is modified by antidepressant treatment. Science 345, 1494-1498. doi: 10.1126/science. 1250469

Shabel, S. J., Proulx, C. D., Trias, A., Murphy, R. T., and Malinow, R. (2012). Input to the lateral habenula from the basal ganglia is excitatory, aversive and suppressed by serotonin. Neuron 74, 475-481. doi: 10.1016/j.neuron.2012. 02.037

Shepard, R. D., and Nugent, F. S. (2020). Early life stress- and drug-induced histone modifications within the ventral tegmental area. Front. Cell. Dev. Biol. 8:588476. doi: $10.3389 /$ fcell.2020.588476

Shepard, R. D., Gouty, S., Kassis, H., Berenji, A., Zhu, W., Cox, B. M., et al. (2018a). Targeting histone deacetylation for recovery of maternal deprivation-induced changes in BDNF and AKAP150 expression in the VTA. Exp. Neurol. 309, 160-168. doi: 10.1016/j.expneurol.2018.08.002

Shepard, R. D., Langlois, L. D., Authement, M. E., and Nugent, F. S. (2020). Histone deacetylase inhibition reduces ventral tegmental area dopamine neuronal hyperexcitability involving AKAP150 signaling following maternal deprivation in juvenile male rats. J. Neurosci. Res. 98, 1457-1467. doi: $10.1002 /$ jnr. 24613

Shepard, R. D., Langlois, L. D., Browne, C. A., Berenji, A., Lucki, I., and Nugent, F. S. (2018b). Ketamine reverses lateral habenula neuronal dysfunction and behavioral immobility in the forced swim test following maternal deprivation in late adolescent rats. Front. Synaptic Neurosci. 10:39. doi: 10.3389/fnsyn.2018.00039

Simmons, S. C., Shepard, R. D., Gouty, S., Langlois, L. D., Flerlage, W. J., Cox, B. M., et al. (2020). Early life stress dysregulates kappa opioid receptor signaling within the lateral habenula. Neurobiol. Stress 13:100267. doi: 10.1016/j.ynstr.2020.100267

Strotmann, B., Heidemann, R. M., Anwander, A., Weiss, M., Trampel, R., Villringer, A., et al. (2014). High-resolution MRI and diffusion-weighted imaging of the human habenula at 7 tesla. J. Magn. Reson. Imaging 39, 1018-1026. doi: 10.1002/jmri.24252

Strotmann, B., Kögler, C., Bazin, P.-L., Weiss, M., Villringer, A., and Turner, R. (2013). Mapping of the internal structure of human habenula with ex vivo MRI at 7T. Front. Hum. Neurosci. 7:878. doi: 10.3389/fnhum.2013. 00878

Sutherland, R. J. (1982). The dorsal diencephalic conduction system: a review of the anatomy and functions of the habenular complex. Neurosci. Biobehav. Rev. 6, 1-13. doi: 10.1016/0149-7634(82)90003-3

Targum, S. D., and Nemeroff, C. B. (2019). The effect of early life stress on adult psychiatric disorders. Innov. Clin. Neurosci. 16, 35-37.

Taylor, S. E. (2010). Mechanisms linking early life stress to adult health outcomes. Proc. Natl. Acad. Sci. U. S. A. 107, 8507-8512. doi: 10.1073/pnas.10038 90107 
Tchenio, A., Lecca, S., Valentinova, K., and Mameli, M. (2017). Limiting habenular hyperactivity ameliorates maternal separation-driven depressive-like symptoms. Nat. Commun. 8:1135. doi: 10.1038/s41467-017-01192-1

Valentinova, K., and Mameli, M. (2016). mGluR-LTD at excitatory and inhibitory synapses in the lateral habenula tunes neuronal output. Cell Rep. 16, 2298-2307. doi: 10.1016/j.celrep.2016.07.064

van Egmond, N., Straub, V. M., and van der Stelt, M. (2021). Targeting endocannabinoid signaling: FAAH and MAG lipase inhibitors. Annu. Rev. Pharmacol. Toxicol. 61, 441-463. doi: 10.1146/annurev-pharmtox-030220112741

Varga, V., Kocsis, B., and Sharp, T. (2003). Electrophysiological evidence for convergence of inputs from the medial prefrontal cortex and lateral habenula on single neurons in the dorsal raphe nucleus. Eur. J. Neurosci. 17, 280-286. doi: 10.1046/j.1460-9568.2003.02465.x

Wallace, M. L., Huang, K. W., Hochbaum, D., Hyun, M., Radeljic, G., and Sabatini, B. L. (2020). Anatomical and single-cell transcriptional profiling of the murine habenular complex. Elife 9:e51271. doi: 10.7554/eLife. 51271

Webster, J. F., Vroman, R., Balueva, K., Wulff, P., Sakata, S., and Wozny, C. (2020). Disentangling neuronal inhibition and inhibitory pathways in the lateral habenula. Sci. Rep. 10:8490. doi: 10.1038/s41598-020-65349-7

Wong, C. A., Ming, D., Maslow, G., and Gifford, E. J. (2020). Mitigating the impacts of the COVID-19 pandemic response on at-risk children. Pediatrics 146:e20200973. doi: 10.1542/peds.2020-0973

Yang, Y., Cui, Y., Sang, K., Dong, Y., Ni, Z., Ma, S., et al. (2018). Ketamine blocks bursting in the lateral habenula to rapidly relieve depression. Nature 554, 317-322. doi: 10.1038/nature25509
Yoshida, T., Fukaya, M., Uchigashima, M., Miura, E., Kamiya, H., Kano, M., et al. (2006). Localization of diacylglycerol lipase-alpha around postsynaptic spine suggests close proximity between production site of an endocannabinoid, 2 arachidonoyl-glycerol and presynaptic cannabinoid CB1 receptor. J. Neurosci. 26, 4740-4751. doi: 10.1523/JNEUROSCI.0054-06.2006

Yoshino, H., Miyamae, T., Hansen, G., Zambrowicz, B., Flynn, M., Pedicord, D., et al. (2011). Postsynaptic diacylglycerol lipase mediates retrograde endocannabinoid suppression of inhibition in mouse prefrontal cortex. J. Physiol. 589, 4857-4884. doi: 10.1113/jphysiol.2011.212225

Zhang, L., Hernández, V. S., Swinny, J. D., Verma, A. K., Giesecke, T., Emery, A. C., et al. (2018). A GABAergic cell type in the lateral habenula links hypothalamic homeostatic and midbrain motivation circuits with sex steroid signaling. Transl. Psychiatry 8:50. doi: 10.1038/s41398-018-0099-5

Zou, S., and Kumar, U. (2018). Cannabinoid receptors and the endocannabinoid system: signaling and function in the central nervous system. Int. J. Mol. Sci. 19:833. doi: 10.3390/ijms19030833

Conflict of Interest: The authors declare that the research was conducted in the absence of any commercial or financial relationships that could be construed as a potential conflict of interest.

Copyright (C) 2021 Shepard and Nugent. This is an open-access article distributed under the terms of the Creative Commons Attribution License (CC BY). The use, distribution or reproduction in other forums is permitted, provided the original author(s) and the copyright owner(s) are credited and that the original publication in this journal is cited, in accordance with accepted academic practice. No use, distribution or reproduction is permitted which does not comply with these terms. 